\title{
Emergence of hierarchy from the evolution of individual influence in an agent-based model
}

\author{
Cedric Perret ${ }^{1}$, Simon T. Powers ${ }^{1}$ and Emma Hart ${ }^{1}$ \\ ${ }^{1}$ School of Computing, Edinburgh Napier University, Edinburgh, EH10 5DT, United Kingdom \\ C.Perret@napier.ac.uk
}

\begin{abstract}
The sudden transition from egalitarian groups to hierarchical societies that occurred with the origin of agriculture is one of the most striking features of the evolution of human societies. Hierarchy is reflected by the evolution of an asymmetrical distribution of the influence of individuals. Although the benefits to leaders themselves are easily justified, it is still hard to identify the causes for the evolution of exploited followers. However, leaders also play an important role in solving coordination problems, a role which would have been amplified by the increase in group size induced by the advent of agriculture. Can this lead to the emergence of leadership directly from the evolution of traits affecting individual influence in group decisions? This question is yet unanswered mainly because of a lack of a mechanistic model linking individual influence to group productivity. Here we fill this gap by explicitly describing the organization of group by a decision-making process. We have developed an evolutionary model where individuals organize to carry out a collective task that produces surplus resources. These surplus resources then drive a demographic expansion of group size. Our results show that a stable distribution of leaders and followers can emerge from the evolution of traits affecting individual influence in decision making, even in the presence of inequality. In addition, our model highlights the conditions and dynamics underlying the development of hierarchy. In line with theoretical work on the evolutionary origins of leadership, this model contributes to understanding the interactions between individual evolution and social structure.
\end{abstract}

\section{Introduction}

Present day societies exhibit highly complex hierarchical structure where a few individuals called leaders have disproportional influence on common decisions. While widespread, hierarchy (also called leadership) has not always been the norm for human groups. Before the first complex societies, small groups of hunter-gatherers were characterized by non-hereditary and facultative form of leadership with a low level of dominance (Boehm, 2001). The social structure of these groups remained largely unmodified for hundreds of thousands of years, with the first hierarchical societies emerging only 12500 years ago during the Neolithic transition from hunter-gathering to agriculture (Price, 1995). Currently widespread, the emergence of leadership is one of the most striking social transitions. While the pervasive presence of hierarchy in current human societies suggests an evolutionary origin, the mechanisms involved in this shift from egalitarian to hierarchical societies are still not completely understood (Van Vugt et al., 2008).

Although leadership may be imposed top-down, e.g. by institutions, it must initially have emerged bottom-up from the evolution of individual behaviour. Hierarchical societies can be defined by a particular distribution of influence among group members, with a minority of highly influential leader individuals, and a majority of influenceable followers (King et al., 2009). The evolutionary basis of leader and follower traits is reflected in heritable psychological traits such as charisma (Judge et al., 2002). The evolution of influence and influencability could thus have been an important first step in the emergence of hierarchy. Yet, it is hard to see how evolution can lead individuals to being influenceable followers, since this an apparently disadvantageous position compared to leaders. Indeed, leaders (thanks to their influence), tend to exploit followers (Hayden and Villeneuve, 2010). To solve this dilemma, the organizational advantage of a hierarchical group is often evoked. Archeological evidence shows that a hierarchical structure could benefit the whole group by reducing the cost of organization (Calvert, 1992), for example in warfare or large-scale construction projects. But despite organizational advantage being an important effect, the investigation of its role in the evolution of leadership has been limited by the absence of models that incorporate an explicit link between individual influence and group organization. To fill this gap, we explicitly model how a group makes a decision, e.g. about where to hunt or how to organise a raiding party. This decision-making process is costly in terms of time - groups that take too long to reach a decision may lose resources or pay other opportunity costs. During the decision-making process, individuals influence each other until they reach a common decision by consensus (Sood et al., 2008). A heterogeneous distribution of influence, with a few leaders and many followers, can lead to faster decision making. However, it can also bias the final decision towards the opinion of the few highly influential individuals 
(Gavrilets et al., 2016). The decision-making process thus links the influence of individuals to the two main mechanisms of leadership: organizational advantage to the group from the time to reach consensus, and individual advantage to the leader from the bias in the final decision.

On the one hand, previous work on decision-making processes has not addressed the evolution of the influence of an individual: the distribution of influence has been assumed to be fixed in models of decision-making. On the other hand, models of the evolution of leadership have never explicitly simulated the decision-making process. Rather, they typically simply assume some organizational benefit to leadership. In this work, we combine a mechanistic model of decision-making with a model of the evolution of a trait affecting individual influence, and use this to answer the following question: Does the evolution of individual influence lead to the emergence of leadership? We find that reducing the time to reach consensus can provide sufficient selection pressure for individuals to evolve into a stable distribution of leaders and followers, even in cases where leaders are despotic.

\section{Background}

Previous work investigating the evolution of leadership has mainly explored two distinct theories. Coercive theories suggest that hierarchy is imposed onto the group by dominant individuals, who would have taken advantage of the advent of food storage and defensible resources to build up resource surpluses that they could then use to control others with (Summers, 2005). But while coercion can explain the maintenance of hierarchical societies, it struggles to explain why some individuals would evolve follower traits and accept an emerging despot in the first place. Indeed, pre-Neolithic human societies had many antiauthoritarian mechanisms inherited from the long period of hunter-gatherer egalitarianism (Boehm, 2001). For instance, the capacity to form a coalition, combined with the invention of projectile weapons, would have made dissatisfied followers a dangerous threat no matter the physical strength of the leader (Bingham, 1999). These ancestral behaviours would have greatly limited the capacity of emerging leaders to impose dominance, implying that coercion alone cannot explain the origin of hierarchy.

On the other hand, functional theories suggest that hierarchy is a mutually beneficial system between leaders and followers (Calvert, 1992). The advent of agriculture and increasing group size brought about new coordination problems, such as the construction of cities, fortifications, and irrigation systems. Evidences suggest that leaders could reduce the cost of organising these tasks, thereby providing a benefit to the whole group. However, this effect alone does not account for the increased levels of despotism that we see during the Neolithic. Why would individuals voluntarily accept a despotic leader?
In reality, both coercive and functional aspects are likely involved in the evolution of leadership (Hooper et al., 2010; Mattison et al., 2016; Powers and Lehmann, 2014). We argue that these aspects of leadership would have both affected, and been affected by, the demographic growth brought about by the Neolithic origin of agriculture (Powers and Lehmann, 2014). The initial demographic growth from agriculture created new large-scale collective tasks, such as constructing irrigation systems and city defences. Although more beneficial, collaboration between a larger number of individuals carries a higher cost of organization (Johnson, 1982). This would have increased the functional benefits of having leaders compared to smaller groups. In turn, groups realising large-scale cooperation thanks to hierarchy would then be able to produce more resources, e.g. through successful irrigation, leading to yet more demographic growth.

Such a feedback loop could have led groups with hierarchy to grow and then expand by migration. In the meantime, other individuals would now require leaders to sustain the organization of their large groups, creating a pressure for them to evolve follower traits. Balanced by this benefit they produce, leaders could have then created a relative level of dominance, e.g. by monopolising a share of the group's resources. In the new world of large-scale collective problems, individuals could still be better off accepting some despotism compared to having no leader at all. Following such a scenario, hierarchy would have emerged as an equilibrium state between the two conflicting roles of leader as producer and despot.

In this paper we use a formal model to determine whether this argument is logically consistent, and to examine the range of conditions under which this feedback process could occur when the decision-making process is explicitly accounted for.

\section{Model definition}

We consider an island model with a population of individuals that is subdivided into a finite number of patches, $N_{\mathrm{p}}$ (Wright, 1931). The life cycle consists of discrete and nonoverlapping generations as follows:

1. Individuals play a decision-making game on their patch, defined below. The time taken to reach consensus is directly translated into an opportunity cost of organization (Equation 7).

2. After consensus is reached, all individuals on a patch take part in a collective task which produces an amount of extra resource, discounted by the cost of organization (Equation 3).

3. The resource obtained from the collective task is distributed among all individuals on the patch as a function of their influence and a parameter $d$ that specifies the level of inequality (Equation 4). 
4. Individuals have a number of offspring drawn from a Poisson distribution, with the mean determined by the resources received (Equations 1 and 2).

5. All individuals of the previous generations perish.

6. Offspring individuals migrate with a probability that results from the sum of a fixed migration rate and an evolved conditional migration rate, which depends on the share of resource their parents received (Equation 8). Migrating individuals suffer a cost $c_{\mathrm{d}}$, and then enter a patch chosen at random from the population (excluding their natal patch).

Individuals are modelled by two traits; their influence $\alpha$, and their intolerance for dominance $z$, both defined between $[0,1]$. The trait $\alpha$ represents the capacity of one individual to modify the opinion of another individual toward its own opinion. At the same time, it influences the probability that an individual talks to other individuals. It represents a set of behavioural traits that modify the influence of an individual, such as talkativeness, boldness, and charisma. A high value of $\alpha$ corresponds to a leader profile, while a low value of $\alpha$ corresponds to a follower profile. A hierarchical structure would be represented by the presence of two subpopulations, a small subpopulation with a high value of $\alpha$, and a large subpopulation with a lower value $\alpha$. For clarity, individuals within these subpopulations will be called, respectively, leaders and followers. The trait $z$ represents the minimum share of resource from the collective action that an individual requires for its offspring to remain on the patch, rather than disperse. As such, $z$ is an individual's intolerance to exploitation by a leader. The two traits $\{\alpha, z\}$ carried by individuals are transmitted vertically from parent to offspring, e.g. by social learning. They mutate following a mutation rate of $\mu_{m}$. As these traits are assumed to be at least partly cultural, the mutation rate is higher than for a classical genetic trait. When a mutation occurs, a random value is sampled from a truncated Gaussian distribution centred on the current value of the trait, with variance $\sigma_{\mathrm{m}}$.

\section{Reproduction}

At each generation, individuals have a number of offspring sampled from a Poisson distribution centred on the individual fitness, $w$. The fitness of individual $i$ on patch $j$ at time $t$ is described by the following equation, where $N_{j}(t)$ is the total number of individual on patch $j$ :

$$
w_{i j}(t)=\frac{r_{\mathrm{a}}}{1+\frac{N_{j}(t)}{K}}+r_{\mathrm{b} i j}(t) .
$$

The fitness of an individual is the sum of an intrinsic growth rate $r_{\mathrm{a}}$ limited by the carrying capacity $K$, and an additional growth rate resulting from the extra resources produced by the collective task, $r_{\mathrm{b} i j}(t)$. The additional growth rate $r_{\mathrm{b} i j}(t)$ is not limited by the carrying capacity, but the competition between individuals is taken into account during the distribution of collective resources. It is calculated as follows:

$$
r_{\mathrm{b} i j}(t)=\beta_{\mathrm{r}}\left(1-e^{-\gamma_{\mathrm{r}}\left(B_{j}(t) p_{i j}(t)\right)}\right) .
$$

The term $r_{\mathrm{b} j}(t)$ is calculated from a logistic function described by $\gamma_{\mathrm{r}}$ and $\beta_{\mathrm{r}}$, respectively the form and the maximum of the increase in growth rate induced by the additional resources. The additional resources are given by the total amount of benefit, $B_{j}(t)$, multiplied by the share the individual receives, $p_{i j}(t)$. The increase of the growth rate follows a logistic relation because of the inevitable presence of other limiting factors of production, e.g. space.

\section{Collective task}

At each generation, the individuals take part in a collective task and produce additional resources $B_{j}(t)$ :

$$
B_{j}(t)=\beta_{\mathrm{b}}\left(1-e^{\left.-\gamma_{\mathrm{b}} N_{j}(t)\right)}\right]\left(1-c_{\mathrm{o} j}(t)\right) .
$$

The benefit is calculated from a logistic function described by $\gamma_{\mathrm{b}}$ and $\beta_{\mathrm{b}}$, respectively the form and the maximum of the increase in the benefit induced by additional participants. The benefit is discounted by the cost of organization, $c_{\mathrm{O}}$. As is standard in microeconomic theory, we make the conservative assumption that the benefit of the collective task has diminishing marginal returns because of other limiting factors of production. To avoid studying the effect of social strategy in the collective task, which has already been extensively studied in the evolution of cooperation literature, we consider that all the individuals on a patch are willing to participate in the collective task. We then focus here on the less studied area of the cost of organization. The collective task simulates the numerous cooperative tasks realized during the lifetime of an individual. It can encompass many actions such as hunting of large game or construction of an irrigation system. The cost of organization comes from the time dedicated to organization instead of realizing the actual task.

\section{Distribution of resources}

The resources produced by the collective task are distributed between the individuals on a given patch. The share of an individual, $p_{i j}(t)$, is then equal to:

$$
p_{i j}(t)=\frac{1+d \alpha_{i j}(t)}{\sum_{i=1}^{N_{j}}\left(1+d \alpha_{i j}(t)\right)} .
$$

The share of an individual is a function of its influence $\alpha$, modulated by a parameter $d$. It is normalized by the share of other individuals within the group. To simulate the dominance of leaders, we consider that individuals use their influence to bias consensus toward their personal interests. In order to keep the model simple, it is not measured from the 
decision making process, but translated into the share of additional resources being directly proportional to the individual influence. The parameter $d$ represents the level of inequality on each patch. For $d=0$, a patch is totally egalitarian and the influence of individuals does not affect the share of each individual. Such a scenario is close to the society of pre-Neolithic hunter-gatherers. For $d=1$, leaders can potentially receive twice what a follower receives. It is assumed for simplicity that $d$ is the same for all patches, and is determined for example by the state of technology, e.g. food storage and military technologies. Nevertheless, different patches can have more or less despotic distributions of resources due to different distributions of $\alpha$ values. In particular, even in a unequal society, the share can be equal if the individuals all have the same influence.

\section{Organization by decision making}

At each generation, the organization is simulated by a decision-making process during which individuals share their opinions until a consensus is reached. The opinions of individuals on the organization of the task is $f$, a continuous value defined on $[0,1]$. The $f$ of each individual is randomly generated at the beginning of the decision-making game. That is because we assume that the tasks tackled by the groups are different at each generation, and so opinions are not heritable. The decision-making game consists of a sequence of discussions between one speaker and a group of listeners. The probability of an individual $i$ on patch $j$ to be chosen as a speaker $s$ is an increasing function of its value $\alpha$ as follows:

$$
\operatorname{Pr}(s)_{i j}=\frac{\alpha_{i j}(t)}{\sum_{i=1}^{N_{j}} \alpha_{i j}(t) .}
$$

The listeners are the other individuals in the group. During a discussion event, the listener update his preference to a value $f_{l}^{\prime}$ following the equation below, where $l$ represents the listener and $s$ the speaker:

$$
f_{l}^{\prime}=f_{l}+\left(\alpha_{s}-\alpha_{l}\right)\left(f_{s}-f_{l}\right) .
$$

When the speaker has a lower $\alpha$ than the listener, the difference is considered still positive but equal to a low value, here 0.01. It also applies when the difference between the speaker and listener $\alpha$ is positive but inferior at this limit. We assume that the position of speaker gives a slight influential advantage over the listeners. This assumption is necessary to avoid a systematic convergence of the preferences toward the individual with the highest $\alpha$, a phenomenon not observed in real life. The individuals play the game until consensus is reached, i.e. the standard deviation of the preferences $f$ is less than $f_{\theta}$. The number of discussion events required to reach consensus is called the time to consensus $t *$. The time to reach consensus is proportional to the cost of organization according to:

$$
c_{\mathrm{o}}=\frac{t *}{C},
$$

where $C$ is a parameter representing the cost of decision making and depends of the limitation of time on the task, for instance, the speed of depletion of resources or the need to build defences before an enemy arrives. We consider here that the final decision reached has no effect on the benefit produced by the collective task. We focus on the general role of hierarchical structure in decision making and not on a trade-off between speed and accuracy, a subject already well examined.

\section{Conditional dispersal of followers}

Additionally to an intrinsic migration rate $m$, offspring also conditionally disperse if the following condition holds:

$$
p_{i j}(t) N_{j}(t)<z_{i j}(t)
$$

where $z_{i j}(t)$ represents the trait of the parent. Similarly to the intrinsic migration, the individuals disperse to a randomly chosen patch (excluding their natal patch). This conditional migration represents the trend of individuals to leave despotic leaders, a potential anti-authoritarian capacity. We assume that individuals evaluate their shares relative to the shares of the other individuals on their patch. Since $p$ is normalized by the size of the group, $z$ is compared to $p N$ to keep the conditional migration independent to the size of the group. The value of $p N$ is equal to 1 into a perfectly egalitarian society, less than 1 if an individuals receive less than a egalitarian share, and higher than 1 if the individual receives more. Because $z$ is defined on [0,1], an individual can leave the group by conditional migration only if it receives less than an equal share. We make the conservative assumption that an individual receiving more than the rest of its group would stay.

The model defines a stochastic process for the two evolving traits $(\alpha, z)$, and for the reproduction and decisionmaking processes. Because of the nonlinearities of the model, which result from the interactions of all of these variables, we analyse it using replicated individual-based simulations.

\section{Results}

The results of interest are the distribution of influence, the mean size of group, and the time to reach consensus. The populations are simulated until an equilibrium state is reached, i.e there are not anymore significant changes in the average value of the variables of interest. In the case of an evolutionary cycle, simulations are performed for a large number of generations in order to determine long-run behaviour of the model. We focus on the effect of the following parameters: (i) the cost of decision-making and (ii) the level of inequality. The default parameters used in the simulations, unless otherwise specified, are $N_{p}=50, N_{j}(0)=10$, $K=20, r_{\mathrm{a}}=2, \beta_{\mathrm{r}}=10, \gamma_{\mathrm{r}}=0.1, \beta_{\mathrm{b}}=2000, \gamma_{\mathrm{b}}=0.01$, 


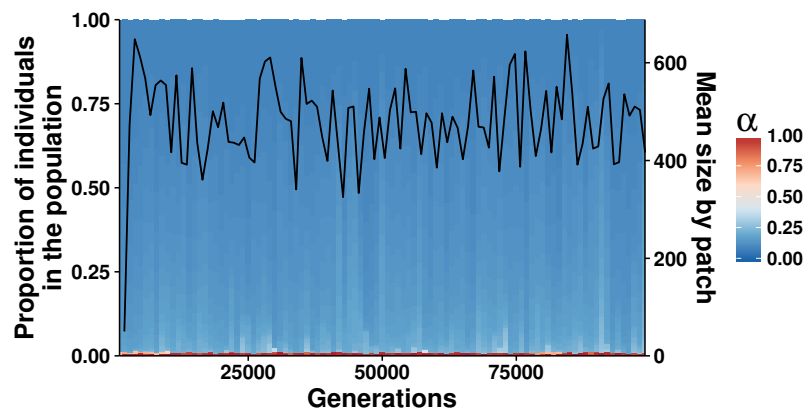

Figure 1: Evolution of the distribution of influence $\alpha$ in the population, and evolution of the mean size of patch, in the absence of inequality $(d=0)$. Parameters : $C=125$

$f_{\theta}=0.01, \mu_{\mathrm{m}}=0.01, \sigma_{\mathrm{m}}=0.01, m=0.2, c_{\mathrm{m}}=0.2$.For each set of parameters considered, 32 independent simulations have been realized. The results presented, unless otherwise specified, are the mean value of replicates and the error bars represent the standard error between the mean value of replicates.

Figure 1 demonstrates that in the absence of inequality $(d=0)$, the evolution of individual influence leads to the emergence of leadership. At the equilibrium state, the distribution of the influence of individuals is divided into two clearly separated subgroups. There are a minority of individuals with high influence (i.e leaders), and a majority of individuals with low influence (i.e. followers). This distribution evolves because groups with a hierarchical distribution of influence reach consensus more quickly during decision-making. Indeed, the few influential individuals quickly bring the opinion of the highly influenceable individuals close to their own opinions. Consequently, a group composed by this heterogeneous distribution pays a low cost of organization and produces a large amount of resources from the collective task. These resources increase the number of offspring of the individuals within the group, which leads to demographic growth as seen in the beginning of the figure 1. Because they grow to a larger size, groups with hierarchy send out a larger number of migrants than groups with less hierarchical distributions of $\alpha$. Ultimately, in the absence of any other evolutionary forces applied to the individual $\alpha$ trait, hierarchical groups propagate this distribution of $\alpha$ until it spreads throughout the global population, leading to the emergence of leadership.

Crucially, as shown by Figure 2, leadership also emerges when the resources are distributed as a function of the influence of an individual, in other words in the presence of inequality. In the long run, the population evolves to a hierarchical distribution with a large part of the population composed of followers with a medium value of influence, and few leaders with extremely high influence. The influ-

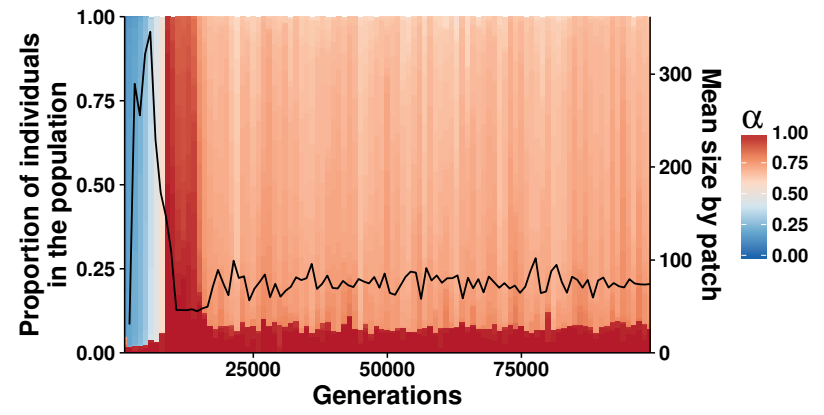

Figure 2: Evolution of the distribution of influence $\alpha$ in the population, and evolution of the mean size of patch, in presence of inequality $(d=1)$. Parameters : $C=125$

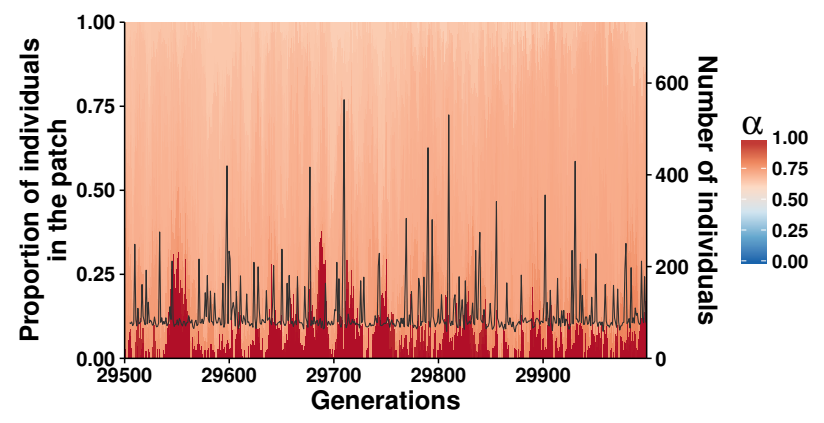

Figure 3: Evolution of the distribution of influence $\alpha$, and evolution of the size of the group on one patch in function of generations in presence of inequality $(d=1)$. Parameters: $C=125$

ence of followers is higher than in the absence of inequality. This leads to less efficient organization through a longer time to reach consensus. Consequently, individuals obtain less resources from the collective action and groups grow to a smaller size. However, this hierarchical distribution still remains stable in the long run.

A first phase can also be observed where the population goes through an initially random distribution of $\alpha$ to a uniform distribution of high $\alpha$. This results from the fact that influential individuals enjoy a fitness advantage because they receive a bigger share of the collective task. During a short period, all of the individuals evolve to have high influence. This leads to costly organization and a small mean patch size. As shown by the long run behaviour of the model, this phase of a uniform high $\alpha$ is only transient and is mainly due to the initial random conditions.

To understand how hierarchy still evolves despite the dominance of leaders, Figure 3 show the evolution of the distribution of influence on a single patch. While the hierarchical distribution is maintained on a global scale, the proportion of leaders and followers within a single patch 
fluctuates. Because the composition of the group impacts the resources produced by the collective task, the size of the group also fluctuates. These cycles directly emerge from the opposing effects of leaders who both give an advantage to the group but also exploit the individuals. Within a group, the more influential individuals receive a larger share of the resources, and so have on average a larger number of offspring. In consequence, selection slowly increases the proportion of leaders on a patch. However, at the same time, the appearance of more influential but also less influenceable individuals (high $\alpha$ ) leads to a slower decision-making process and reduces the total benefit received from the collective task.

At some point, the proportion of leaders becomes too high and the cost of organization cancels the benefit from resources produced by the collective task. It also means that the influential individuals no longer receive a bigger share of the resources. Hence, within the group, the previous selection pressure leading to the increase of the proportion of leaders disappears and the values of influence $\alpha$ randomly varies because of the demographic stochasticity. However, in the meantime, some groups present in other patches are successfully organizing collective tasks thanks to their more marked hierarchical structure. Being larger than the groups where hierarchy collapsed, they consequently produce a higher number of migrants. These migrants are mainly followers, firstly because they formed the majority of the population source and secondly because conditional migration led dissatisfied followers to other patches. This process leads to the re-emergence of hierarchical structure and then the reappearance of the resources produced by the collective task. In turn, this now hierarchical group grows larger and sends follower migrants to other groups. This hierarchical structure would then be maintained until the selection due to dominance restarts the cycle.

Figure 4(a) illustrates the mechanisms behind the cycles by highlighting the role of $C$, the cost of decision-making. The results demonstrate that the followers evolve a mean of $\alpha$ proportional to $C$. This is because $C$ represents the limit at which collective action succeeds or fails, in other words the limit at which selection leads or not to an increase in the proportion of leaders. For a more costly decision-making process, the asymmetrical distribution of resources only appears in a group with efficient hierarchy, i.e. when the followers are more influenceable (lower $\alpha$ ). In the same time, the cost of decision-making means that fewer groups at a time get to the point of producing resources from the collective task, which leads to a smaller global population. In the case of hierarchy collapsing synchronously in all patches, the dynamic previously described reappears quickly by genetic drift. As illustrated by Figure 4(b), the value of the time to consensus then oscillates around the value of $C$. On the other hand, when the cost of decision-making is too low $(C=400)$, the advantage of hierarchical distribution is too
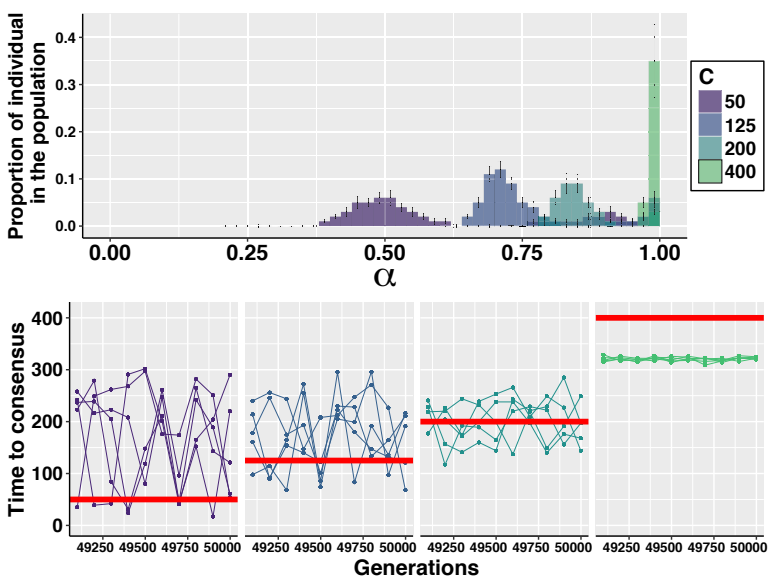

Figure 4: (a) Distribution of the influence $\alpha$ in the population after 50000 generations for different costs of decisionmaking $C$. (b) How the time to reach consensus varies across generations at the equilibrium state (after the simulation has already been run for 49000 generations). Although qualitatively robust across all patches, only 5 patches are represented for clarity. The value of $C$ is represented by the horizontal red line. Parameters : $d=1$

small to maintain leadership in the population. There are then no groups who reach the limit at which collective action fails and the competition between individuals leads their evolution toward a high $\alpha$.

In addition, the results show greater fluctuations of the time to consensus for low values of $C$. That is because at the population scale, fewer groups have efficient hierarchy and so the absolute number of follower migrants with low influence is smaller. Consequently, the groups where hierarchy is not marked enough to produce any resources take more time to return to a more efficient distribution of influence. In the opposite situation, when decision-making is not costly, many hierarchical groups are present at the same time sending a large number of migrants, which will quickly reintroduce an efficient hierarchy within the groups. It is worth noting that the individuals rarely reach a time to consensus much lower than $C$ and so on average have a relatively poor organization. This effect is due to the dominance of leaders which leads to smaller groups than in a population where inequality is absent.

Figure 5 shows that a higher level of inequality $d$ leads to the evolution of a higher mean of influence $\alpha$ of the followers. These results are explained by the role of the level of inequality which directly affects the advantage of influential individuals. A high level of inequality leads to a strongly asymmetric distribution of resources. Because the influential individuals receive a large amount of resources, it leads to a rapid increase in the proportion of leaders and groups 


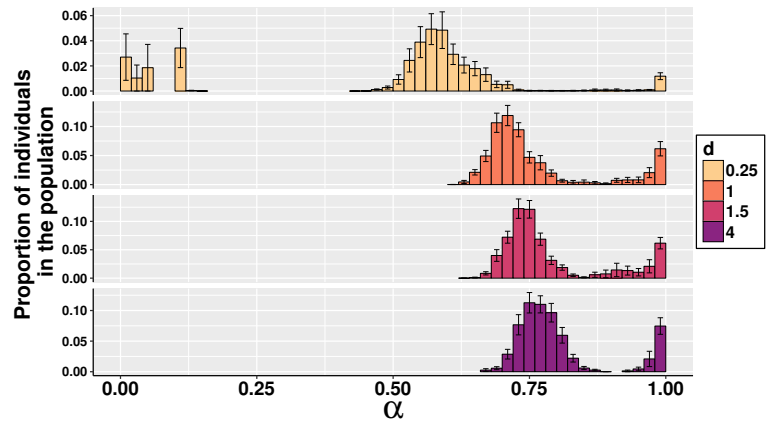

Figure 5: Distribution of the influence $\alpha$ in the population after 50000 generations for different level of inequality. Parameters: $C=125$
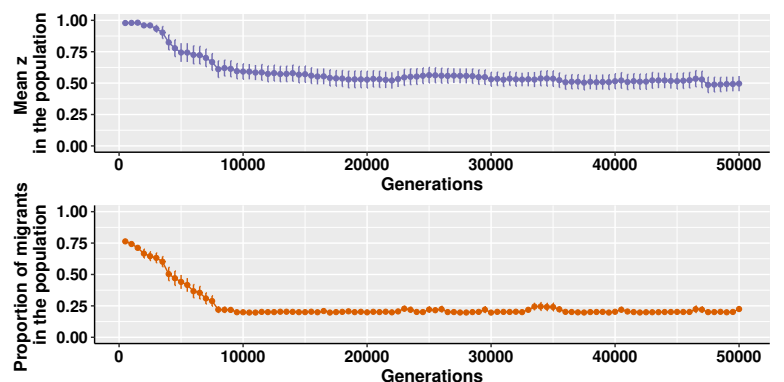

Figure 6: (a) Evolution of the intolerance to despotism $z$ in the population. (b) Evolution of the proportion of migrants in the population. Parameters: $C=125, d=1$

more quickly reach the point where consensus takes so long that the collective task does not provide any resources. Consequently, on average, fewer groups have a functional hierarchy with followers of low $\alpha$. It is worth noting that hierarchy is still present even for relatively high level of inequality $(d=4)$. When the level of inequality is low $(d=0.25)$, the results show that in few simulations, the population evolves to a really marked hierarchical distribution. In such case, the migration from efficient groups has been enough to balance the individual advantage of leaders.

Figure 6(a) shows that $z$, the intolerance to despotism, evolves to a relatively medium fixed value. Interestingly as shown by the figure $6(\mathrm{~b})$, the proportion of migrants remains stable (around 0.25) over time. The evolution of $z$ is constrained by the cost of migration on one hand and the competition within the patch on the other. In particular, the followers evolve a $z$ to counter balance the exploitation by the leaders. This can be seen during the first thousand generations corresponding to the transient phase where individuals still have a high intolerance to despotism $z$ in response of the invasion of highly influent but also dominant individuals within the groups. The low value of $z$ during the equilibrium state is because the advantage of leaving the group is higher than the cost to migrate only when the individuals are too heavily exploited. Although limited, it maintains hierarchy in the population by sending influenceable followers to other patches.

\section{Discussion}

The origin of leadership in human societies is still hard to comprehend. In particular, leaders tend to exploit followers which should theoretically limit their evolution. Although often described as an asymmetrical distribution of influence, the emergence of hierarchy and increasing group size via the evolution of individual influence has not yet been investigated. We have simulated such a scenario by explicitly describing the decision-making process required to perform any collective task. Our results demonstrate that evolution can lead to the emergence of a stable hierarchy with a minority of influential individuals as leaders and a majority of influenceable individuals as followers. Within a patch, groups fluctuate between efficient coordination thanks to hierarchy and collapse of collective task solving because of internal conflicts, in which individuals try to become leaders in order to obtain more resources for themselves. Despite this, leadership is stable in the global population.

These results are in line with previous theoretical work showing that the negative effect of a leaders dominance can be balanced by the organizational advantage it provide (Hooper et al., 2010; Powers and Lehmann, 2014). The model developed by Powers et al shows that leadership as an institution can evolve if hierarchy leads to an increase in group size (Powers and Lehmann, 2014). Our model has independently confirmed that this prediction holds even when leadership emerges bottom-up from the evolution of traits affecting individual influence. Moreover, our model shows that the benefit of hierarchical groups can emerge from a simple and general low-level mechanism, the decisionmaking process. To understand whether leadership could have emerged first from bottom-up individual behaviour and then later become institutionalised, or whether the opposite is true, is still an important question to fully understand the evolution of leadership. This model shows that the former is a plausible scenario and suggests further investigation of the interactions between bottom-up and top-down creation of leadership roles in societies.

Our model predicts that although stable on a global scale, societies should follow cycles in their size and in their social organization. Such behaviour of societies may relate to the succession of efficient hierarchy and internal conflicts between leaders, as observed during human history (Diamond, 2005). It is worth noting that these cycles also lead to poorly functional hierarchy in which individuals rarely organize optimally. However, it is more probable that in real societies, other mechanisms not implemented here would limit the evolution of increasing numbers of leaders within 
a group, leading to more efficient hierarchy. For instance, anti-authoritarian mechanisms inherited from an egalitarian period as well as competition between leaders could strongly limit the advantage of followers trying to become leaders.

The results predict that a more costly task will lead to a more marked hierarchy better at organizing but also to a larger number of groups where organization is insufficient to produce resources. This is reflected in the tendency of groups to shift to important hierarchy in time-limited task such as warfare. Finally, the model suggests that although inequality is often evoked as a main threat to leadership, hierarchical distribution of influence can evolve even under high levels of inequality.

The model allowed us to identify the two main mechanisms behind these predictions. First, the increase in the proportion of leaders provokes the collapse of collective tasks but does not lead to a complete disappearance of hierarchy. This is because influential individuals only receive more resources from the collective task and not from the personal resources of each individual. In the same manner, empirical evidence shows preferential access of leaders to resources, but also suggests that access is limited to the collective resources (Hayden and Villeneuve, 2010). Indeed, the important anti-authoritarian system of pre-Neolithic populations greatly limited the exploitation of personal resources (Boehm, 2001). In the absence of institutions to impose a leaders coercion, it seems then more plausible that the leaders receive a larger share of resources via their influence in decision making processes. Although not taken into account here for simplicity reasons, this effect appears in decisionmaking where the value of the final decision is biased toward the values of influent individuals (Gavrilets et al., 2016). Secondly, the migration from large hierarchical groups helps in maintaining or reintroducing leadership in other groups. This effect is explained by the more efficient organization of the groups with a hierarchical distribution of influence. Our results confirm previous work where groups with a heterogeneous distribution of influence perform better in decisionmaking (Gavrilets et al., 2016; Sood et al., 2008). On a more top-down view, the role of leaders in solving coordination problems have been illustrated by much anthropological evidence (Carballo et al., 2014).

The model developed here has demonstrated that the emergence of leadership from the evolution of individual influence is plausible. It predicts that transition from egalitarian to hierarchical societies will happen when (i) hierarchical groups generate more resources leading to their demographic expansion; (ii) the dominance of leaders is limited to the public good resources produced through collective action. In addition, our model demonstrates that the decision-making process successfully captures the effects of leadership and links individual behavioural traits to group dynamics.

\section{References}

Bingham, P. M. (1999). Human uniqueness: a general theory. The Quarterly Review of Biology, 74(2):133-169.

Boehm, C. (2001). Hierarchy in the forest: the evolution of egalitarian behavior. Harvard University Press, Cambridge.

Calvert, R. (1992). Leadership and its basis in problems of social coordination. International Political Science Review, 13(1):7-24.

Carballo, D. M., Roscoe, P., and Feinman, G. M. (2014). Cooperation and collective action in the cultural evolution of complex societies. Journal of Archaeological Method and Theory, 21(1):98-133.

Diamond, J. M. (2005). Collapse: how societies choose to fail or succeed. Viking, New York.

Gavrilets, S., Auerbach, J., and Van Vugt, M. (2016). Convergence to consensus in heterogeneous groups and the emergence of informal leadership. Scientific Reports, 6(January).

Hayden, B. and Villeneuve, S. (2010). Who benefits from complexity? A view from Futuna. pages 95-145. Springer New York.

Hooper, P. L., Kaplan, H. S., and Boone, J. L. (2010). A theory of leadership in human cooperative groups. Journal of Theoretical Biology, 265(4):633-646.

Johnson, G. A. (1982). Organizational structure and scalar stress. In Theory and Explanation in Archaeology, pages 389-421.

Judge, T. A., Bono, J. E., Ilies, R., and Gerhardt, M. W. (2002). Personality and leadership: A qualitative and quantitative review. Journal of Applied Psychology, 87(4):765-780.

King, A. J., Johnson, D. D. P., and Van Vugt, M. (2009). The origins and evolution of leadership. Current Biology, 19(19):R911-R916.

Mattison, S. M., Smith, E. A., Shenk, M. K., and Cochrane, E. E. (2016). The evolution of inequality. Evolutionary Anthropology, 25(4):184-199.

Powers, S. T. and Lehmann, L. (2014). An evolutionary model explaining the Neolithic transition from egalitarianism to leadership and despotism. Proceedings of the Royal Society B: Biological Sciences, 281(1791):20141349-20141349.

Price, D. T. (1995). Social inequality at the origins of agriculture. In Price, T. and Feinman, G., editors, Foundations of social inequality, chapter 5, pages 129-151. NY: Plenum Press, New York.

Sood, V., Antal, T., and Redner, S. (2008). Voter models on heterogeneous networks. Physical Review E - Statistical, Nonlinear, and Soft Matter Physics, 77(4):041121.

Summers, K. (2005). The evolutionary ecology of despotism. Evolution and Human Behavior, 26(1):106-135.

Van Vugt, M., Hogan, R., and Kaiser, R. B. (2008). Leadership, followership, and evolution: Some lessons from the past. American Psychologist, 63(3):182-196.

Wright, S. (1931). Evolution in mendelian populations. Genetics, 16(2):97-159. 